\title{
External Ventricular Drains: An Overview of Indications, Technique, and Complications
}

\author{
Anudeep Yekula ${ }^{1}$, Aditya Mittal $^{2}$, Varun Sagi ${ }^{3}$ and Mihir Gupta ${ }^{4 *}$ \\ ${ }^{1}$ Department of Neurosurgery, Massachusetts General Hospital, Boston, MA, United \\ States of America \\ ${ }^{2}$ School of Medicine, University of Pittsburgh, Pittsburgh, PA, United States of America \\ ${ }^{3}$ School of Medicine, University of Minnesota Twin Cities, Minneapolis, MN, United \\ States of America \\ ${ }^{4}$ Department of Neurosurgery, University of California San Diego, La Jolla, CA, United \\ States of America \\ *Corresponding Author: Mihir Gupta, Department of Neurosurgery, University of \\ California San Diego, La Jolla, CA, United States of America.
}

\author{
Received: May 28, 2020 \\ Published: June 30, 2020 \\ (C) All rights are reserved by Mihir Gupta., \\ et al.
}

\begin{abstract}
External ventricular drains (EVDs or ventriculostomies) are frequently required for acute management of intracranial hypertension and monitoring intracranial pressure. Patients with ventriculostomies are frequently co-managed by multiple specialists including neurologists, neuro-intensivists, trauma specialists, emergency department physicians and neurosurgeons. We provide background on the indications, placement technique, troubleshooting and complications related to this procedure. We also discuss emerging best practices for preventing complications such as ventriculostomy-related infection. We conclude with considerations regarding ventriculostomy removal and areas of ongoing study. This review serves as a primer that may facilitate collaborative care between diverse allied providers.

Keywords: External Ventricular Drain; EVD; Ventriculostomy; Kocher's Point; Hydrocephalus; Cerebrospinal Fluid; Neurocritical Care; Intracranial Pressure; Ventriculitis; Intracranial Hemorrhage
\end{abstract}

\section{Abbreviations}

CSF: Cerebrospinal Fluid; EVD: External Ventricular Drain; ICH: Intracranial Hematoma; ICP: Intracranial Pressure

\section{Background}

External ventricular drains (EVDs), also known as extraventricular drains or ventriculostomies, are one of the most widely performed cranial neurosurgical procedures [26]. EVDs are used in the emergent management of life-threatening acute hydrocephalus and intracranial hypertension secondary to pathologies ranging from intracranial hematoma (ICH) to infections, neoplasms, ventriculoperitoneal shunt failure and traumatic brain injury (TBI) [13]. Patients with EVDs are frequently managed by teams of diverse specialists. In-depth evaluation of the myriad clinical questions surrounding EVDs is challenged by highly variable practice patterns, as well as the limited quantity and quality of available evidence. Nonetheless, there remains a critical need for reviews that outline practical considerations and provide a common vocabulary to guide patient management by diverse provider teams. Accordingly, we describe the indications, placement technique, complication avoidance methods and emerging best practices related to this procedure, with the goal of facilitating interdisciplinary dialogue and patient care.

\section{Indications for EVD placement}

The two broad indications for EVD placement are to monitor ICP and divert cerebrospinal fluid (CSF) [13]. Intracranial hypertension can occur in a variety of pathologies and is defined as sustained ICP greater than $15-25 \mathrm{~mm} \mathrm{Hg}$, though the exact threshold varies between different studies. Recognizing signs of elevated ICP and tailoring treatment to the underlying cause remain critical to preventing sequelae such as cerebral hypoperfusion, herniation and brain death. Initial management is often with medical measures such as head elevation, intravenous hyperosmolar therapy and sedation. Control of fevers, seizures and metabolic derangements is also critical. Endotracheal intubation, transient hyperventilation or pharmacologic neuromuscular paralysis may be necessary in medi- 
cally refractory cases. Although CSF comprises only approximately $5 \%$ of the intracranial volume, patients with impaired cerebral autoregulation may experience exponential changes in ICP with even small changes in volume. Furthermore, CSF diversion may also aid in clearing harmful inflammatory mediators that contribute to the pathophysiology of acute cerebral injury [1].

In the updated fourth edition of management guidelines for traumatic brain injury (TBI), the Brain Trauma Foundation provides several recommendations regarding the indications for ICP monitoring and CSF diversion. Notably, all recommendations on these topics are designated level IIB or III, indicating low-quality published evidence. ICP monitoring is advised in all patients with an abnormal CT scan presenting with severe TBI, defined as postresuscitation Glasgow Coma Score (GCS) between 3 and 8, in order to reduce in-hospital and subacute mortality. ICP monitoring may be considered in patients with severe TBI and normal CT scans if at least two of the following are noted at presentation: systolic blood pressure $<90 \mathrm{~mm} \mathrm{Hg}$, motor posturing, or age above 40 years. Continuous CSF drainage with an appropriately calibrated EVD system was noted to mitigate intracranial hypertension and may be considered within the first 12 hours after injury in patients presenting with GCS $<6[6]$.

Acute EVD placement can serve as a critical temporizing measure while definitive neurosurgical intervention is planned for relief of mass effect from space-occupying lesions, hematomas, fractures and other life-threatening pathologies. EVDs may eventually be converted to implanted ventriculo- or lumbo-peritoneal shunts for patients who do not recover cerebral autoregulation [8]. In addition, EVDs may be required for temporary CSF diversion during treatment for infected shunts.

\section{Anatomy}

The most common entry point for EVD insertion is Kocher's point on the right side, located $1 \mathrm{~cm}$ anterior to the coronal suture and $2-3 \mathrm{~cm}$ lateral to midline. In most adults, this corresponds to a point along the mid-pupillary line and $11-12 \mathrm{~cm}$ posterior to the nasion [9]. Entry via the non-dominant right frontal lobe is preferred to minimize the risk of damage to language function. Staying anterior to the coronal suture ensures sufficient distance from eloquent cortical and subcortical motor regions. Left side Kocher's point and occipital trajectories are less commonly practiced in adults.

The target for EVD placement is the ipsilateral foramen of Monro, a conduit between the lateral and third ventricles. Multiple structures traverse the foramen, including the thalamostriate, septal and superior choroidal veins, as well as the choroid plexus and branches of the medial posterior choroidal arteries. The foramen is related to the thalamus posteriorly and the fornix anteriorly [28]. The frontal horn of the lateral ventricle, meanwhile, is encircled by the corpus callosum inferiorly, anteriorly and superiorly. The medial and lateral walls are formed by the septum pellucidum and head of the caudate nucleus, respectively (Figure 1). Misplacement of the EVD catheter risks damage to any of the multiple aforementioned critical structures; these complications are further discussed below.

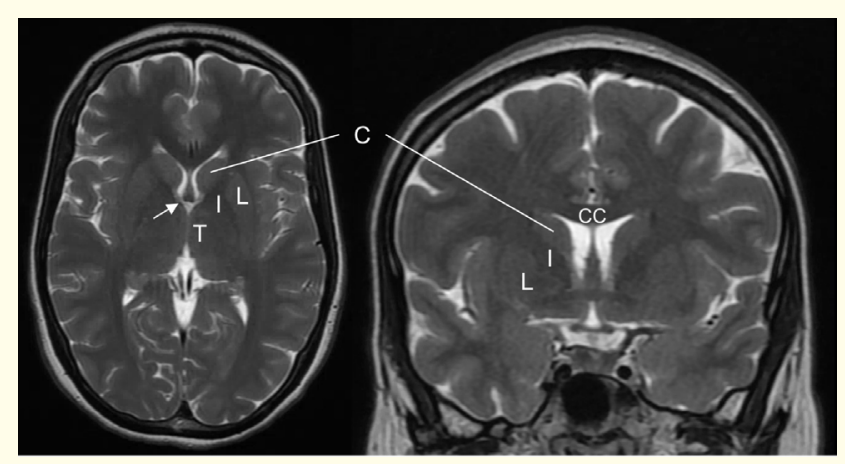

Figure 1: Non-contrast magnetic resonance images of the brain in the axial (left image) and coronal (right image) planes shows the relationships of the caudate nucleus (C), internal capsule (I), lentiform nucleus (L), thalamus (T), corpus callosum (CC) and foramen of Monro (arrow).

Technique for free-hand EVD placement at Kocher's point

A pre-procedural 'time-out' should be conducted to identify the patient, allergies, procedure, side, antibiotic prophylaxis, blood products, laboratory values, local anesthetic, sedation, and appropriate supplies (Listed in box X). A broad area of the correct side of the head is shaved; in our practice we shave the frontal area extending posteriorly until the parietal boss and medially until just across midline.

The patient is positioned supine, with the top of the head flush with the top of the gurney mattress; this may require carefully moving the patient towards the head of the bed. The head of the bed is elevated and the head firmly secured in neutral position with tape. Markings are made at midline and Kocher's point. Following injection of local anesthetic at the planned incision site, sterile preparation and draping is performed. A short linear incision is made at Kocher's point and the skin edges are held with a small retractor. A bur hole is made using a hand drill. The dura is opened sharply with a needle or trocar.

The trajectory of the catheter is then confirmed (Figure 2). In the coronal plane, the catheter is generally aimed towards the ip- 


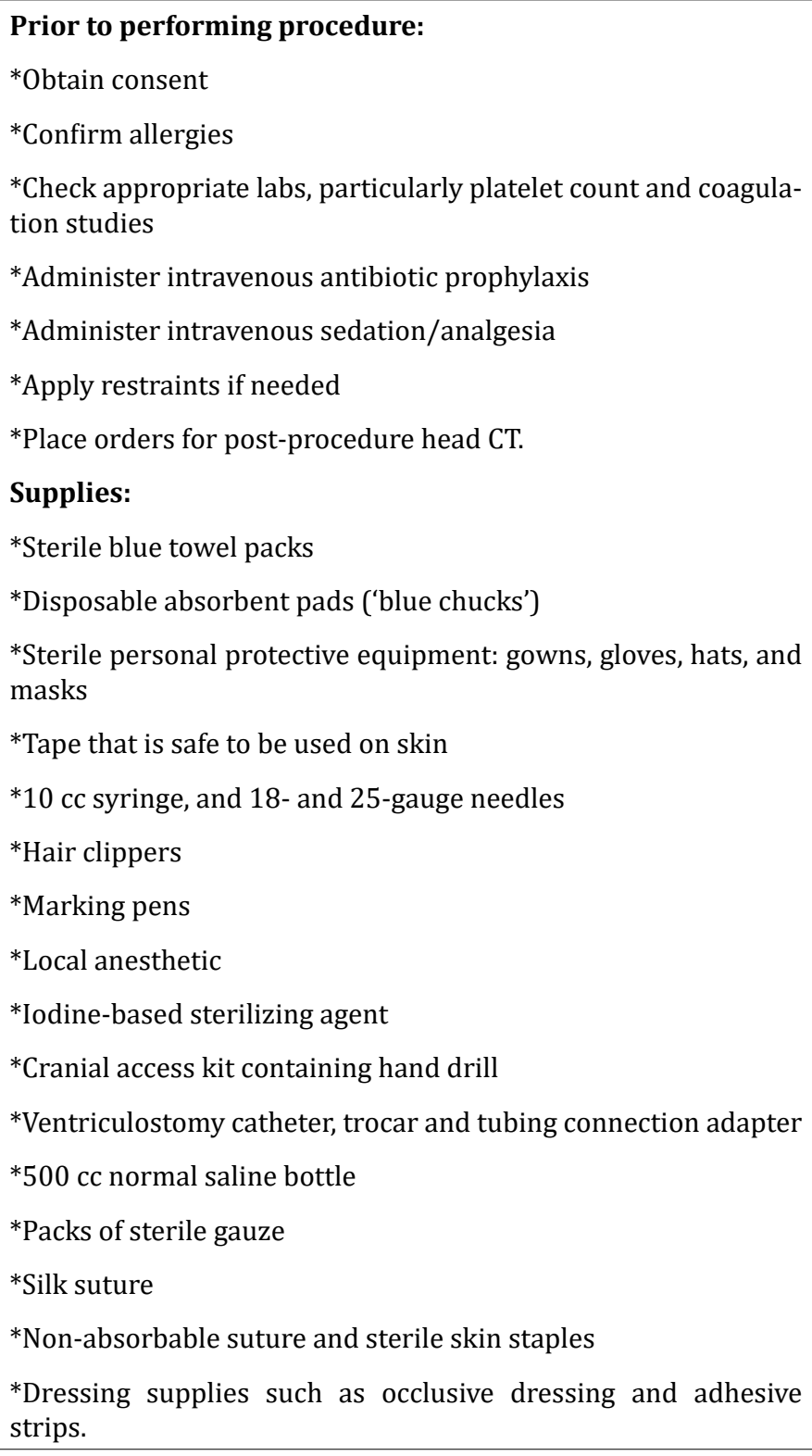

Box X: Ventriculostomy supply checklist.

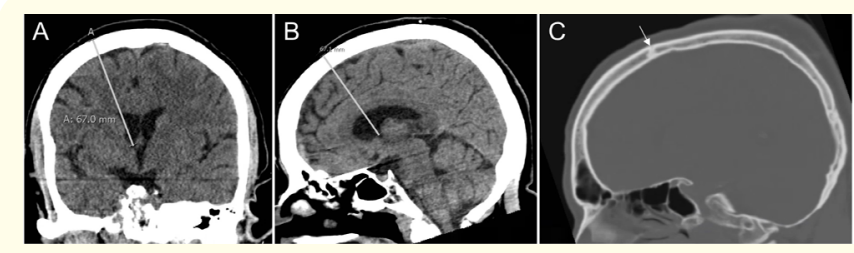

Figure 2: Non-contrast computed tomography scan of the head in the coronal (A) and sagittal (B) planes shows the planned trajectory of a right frontal ventriculostomy catheter, from the outer skull surface to the ipsilateral foramen of Monro. (C) A sagittal image with bone windowing from a separate patient shows the coronal suture (arrow). silateral medial canthus. In the sagittal plane, one may aim for a point one-third of the way from the tragus to the lateral canthus. One may place a cylindrical object such as a plastic sheath or syringe tubing against the skull in order to visualize a perpendicular trajectory and then make small adjustments to aim accurately at the aforementioned landmarks.

The ventriculostomy catheter, with its stylet in place, is then passed through the bur hole until the ventricle is entered. In adults, this distance is usually around $4-5 \mathrm{~cm}$, measured from the outer skull surface. The stylet is then carefully withdrawn while holding the catheter in place. If cerebrospinal fluid (CSF) flow is appreciated, the catheter is then passed to the final depth gently by hand, without replacing the stylet. The catheter is then attached to a trocar and tunneled out through the skin within the sterile field. The exit site should be at least $4 \mathrm{~cm}$ away from the incision. CSF flow is again checked, and the catheter is then secured at the exit site with non-absorbable suture. In case CSF flow is not obtained after catheter insertion, there are a variety of troubleshooting strategies one may consider (Described in box Y).

In case cerebrospinal fluid is not obtained upon passing the ventriculostomy catheter, consider one of the following maneuvers:

*Double-check measurements, landmarks and trajectory planning. It often helps to have an independent observer verify the landmarks while standing in a different location than the individual performing the procedure. Most commonly the bur hole is placed too anteriorly and/or too medially. Carefully remove the catheter, flush with copious sterile saline, drill a separate bur hole (if appropriate) and then reinsert the catheter. Multiple insertion attempts should generally be avoided in order to minimize the risk of tissue damage or hemorrhage.

*If additional insertion attempts are deemed unsafe, consider placing a fiberoptic ICP monitor in lieu of a ventriculostomy. This may serve as a temporizing measure that provides accurate ICP measurements while further troubleshooting can be undertaken. If this is anticipated, one should include the possibility of ICP monitor placement in the procedural consent and discuss the risks and benefits accordingly.

*One may consider leaving the ventriculostomy catheter in place and getting a CT scan. This can reveal adjustments that need to be made to the catheter trajectory, or factors such as hematoma that preclude successful placement.

Box Y: Intra-procedural troubleshooting.

At this point, the catheter may be connected to the collection chamber and pressure transducer, in order to confirm CSF flow and appropriate ICP waveform, respectively. The opening pressure should be recorded in both nursing and physician documentation. The catheter is then secured to the skin and the incision is closed; 
either non-absorbable sutures or sterile skin staples may be used for these purposes. Finally, the area is dressed in accordance with institution-specific protocols (see below for further discussion of this topic).

After removing supplies and repositioning the patient, ensure that the collection chamber is leveled to the tragus, set to the correct height, and flowing appropriately. A non-contrast head CT scan should be obtained as soon as feasible post-procedurally. Although there is no substitute for independent repetition to master any procedure, we share several pearls from our own experience in box Z.

*Rigorously optimize patient positioning. The head should be at a comfortable height for the operator, usually at the level of the navel or lower chest. Introduce flexion at the knees to minimize the chance of the patient sliding toward the foot of the bed during the procedure.

*Avoid excess flexion of the patient's neck in order to maximize venous drainage, minimize intra-procedural bleeding, and reduce intracranial pressure. In general, there should be at least two finger breadths of space between the chin and sternum.

*Optimize the room setup. Pull the gurney forward to create space for the operator to stand and maneuver comfortably at the head of the bed. Set up at least two mobile stands to ensure sufficient working area. Place a large garbage can within the operator's reach.

*Make sure wires, tubes, monitors and equipment such as ventilators are safely positioned away from the working area. The monitor displaying vitals and ICP waveform should be visible to the operator at all times. A separate monitor displaying the CT scan and planned measurements should also be visible.

*Ensure that the nursing team is comfortable setting up the collection chamber and pressure transducer, so this can be performed while the procedure is under way. Alternatively, set up the chamber and transducer before starting the procedure.

*Double-check the depth of the catheter at the outer skull surface after tunneling, and also immediately before closing the incision. In case any deviations from the planned depth are noted, carefully make the necessary adjustments and re-check CSF flow.

*Ensure that all necessary supplies are stocked and regularly checked in all hospital sites where ventriculostomies are commonly performed (such as the emergency department, intensive care unit and operating rooms). Consider providing the physician and nursing teams in each department with a supply checklist. Some units may also maintain a box or supply cart with all necessary supplies.

Box Z: Ventriculostomy placement pearls.

Procedural complications and troubleshooting

The most common complications related to EVD placement include ventriculostomy-related infections, intracranial hemorrhage, neurological deficit due to catheter malposition and catheter occlusion. Studies of each complication suffer from inconsis- tent defining criteria, widely variable practice patterns, and a lack of prospective or randomized studies to establish incidence and guide management. Collaborative clinical teams must thus engage in interdisciplinary discussion to develop protocols for diagnosing and preventing complications that are compatible with local practice and provider preferences. This requires first rigorously reviewing institutional experience with various complications, ideally in the setting of a forum such as a regular multidisciplinary morbidity and mortality conference. Protocols should then be standardized as much as possible, guided by the literature. The use of 'care bundles' to standardize management practices is further discussed in the final section of this report.

\section{Ventriculostomy-related infections (VRIs)}

Extensive criteria for diagnosing and managing healthcareassociated ventriculitis and meningitis have been proposed by groups including the Infectious Diseases Society of America [29] and Centers for Disease Control [15]. CSF culture remains the most important modality to establish the diagnosis of VRI, particularly in conjunction with CSF pleocytosis, hypoglycorrhachia and/or clinical signs of ventriculitis or meningitis. In patients with suspected VRI and negative initial culture results, cultures should be held for at least 10 days in order to detect indolent organisms such as $P$. acnes. The most common bacterial organisms include coagulasenegative Staphylococci, Staphylococcus aureus, Acinetobacter species and polymicrobial infections [11].

However, culture results should be interpreted with caution due to the possibility of contaminants and colonizing organisms that do not cause infection [12]. Furthermore, CSF chemistries and cell counts abnormalities may overlap significantly between infection and phenomena such as hemorrhage, trauma and neurosurgery that are common in patients with EVDs. Additionally, there is no gold-standard reference biomarker of VRI [11]. Consequently, there remains no widely accepted definition of VRI. Studies have thus used highly variable criteria, leading to estimates of infection rates ranging widely from $0 \%$ up to $32 \%[7,11,20,22]$.

Variability in definitions and study methodology also complicate attempts to study other critical questions. For example, while common sense supports a positive correlation between infection risk and length of time the catheter is in place, the evidence for this relationship is mixed $[2,12,18,21]$. Similarly, randomized trials of antibiotic prophylaxis given peri-procedurally versus for the duration of drain placement have been subject to sample size and methodological issues such as giving different antibiotics to patients in different study arms [5,23]. More robust randomized trial evidence exists to support the use of antibiotic-impregnated EVD catheters $[24,30]$, but with variable extent of benefit in terms of reducing VRI 
rates. Common practice in many large centers, supported by Neurocritical Care Society guidelines, is to give one only dose of prophylactic antibiotics peri-procedurally, use antibiotic-impregnated catheters, and remove EVDs as soon as clinically feasible. Previously explored practices such as routine catheter site changes are now also recommended against [12].

\section{EVD-related hemorrhage}

Studies of post-procedural hemorrhage have followed largely the same themes as those pertaining to VRIs. The definition of hemorrhage is highly variable, and consequently reported rates have ranged from $0 \%$ to $41 \%[3,4,10]$. In general, hemorrhage rates are likely closer to those found by Kakarla., et al. who noted an overall rate of $5 \%$, which were predominantly small tract hemorrhages $(3.8 \%)$ and in some cases extra-axial or intraventricular $(1.5 \%)$. The majority of hemorrhages are asymptomatic, but 0.7 - $2.4 \%$ may be clinically significant $[12,16]$. Symptoms may include neurologic deterioration, focal deficit, seizure or symptoms related to mass effect and intracranial hypertension. Risk factors for procedural hemorrhage remain unclear, but may include coagulopathy, hypertension and multiple insertion attempts. However, thresholds for coagulation function have not been standardized and are unlikely to be studied in randomized trials. Prevailing clinical practice and guidelines thus advise correcting coagulopathy and controlling parameters such as blood pressure and the number of passes during insertion as much as clinically feasible [12].

\section{Catheter occlusion}

Although not rigorously studied in the literature, EVD catheter occlusion is among the most frequent daily obstacles encountered in clinical practice. This manifests as a reduction or cessation of CSF output, and often a loss of accurate ICP measurement. Patients may also develop signs of intracranial hypertension if catheter patency is not restored. Risk factors for occlusion have not been studied to our knowledge, but may include intraventricular hematoma or debris, such as from neoplasms or advanced intracranial infections. Collapsed ventricles with minimal CSF volume and mechanical obstruction of catheter inlet holes may also obstruct catheters. Mal-positioned catheters may also be more prone to obstruction. Rare causes include kinked, damaged or disconnected catheter tubing.

Occlusion may be diagnosed by carefully lowering the collection chamber to confirm the loss of flow. Careful inspection of the external catheter, from its exit site until the chamber, is also critical. CT scan may be considered to exclude structural factors such as new intracranial hematoma.

The catheter may be flushed with sterile saline under strict sterile conditions in order to flush out obstructing material. It is in general agreed that flushing distally (towards the collection chamber) is safer than flushing proximally (into the ventricles); the latter should be avoided whenever possible and only performed by experienced providers with extreme caution. Importantly, the amount of fluid flushed into the collection chamber should be monitored and excluded from the CSF output tabulation. Ultimately, catheter replacement may be required as a last resort.

\section{Criteria for EVD removal}

Removal of EVDs is most commonly performed by a gradual 'weaning' strategy and a 'clamp trial'. This involves raising the height of the drain by $5 \mathrm{~cm}$ every day until a level of $20-25 \mathrm{~cm}$ is reached. The drain is then clamped and the patient monitored for 24 - $48 \mathrm{hrs}$. Reopening of the EVD may be required if the ICP exceeds $20 \mathrm{~mm} \mathrm{Hg}$ for over 5 - 10 minutes, clinical deterioration occurs, or symptoms of intracranial hypertension develop. CT scans are obtained before and after the clamp trial; removal may be contraindicated if ventricular enlargement or other signs of hydrocephalus develop.

Failure to wean an EVD requires placement of a ventriculoperitoneal shunt and/or performance of endoscopic third ventriculostomy, depending on clinical factors and provider preference. Although most providers still gradually raise the EVD height before attempting a clamp trial, two studies suggest that alternate approaches may be safe. Klopfenstein., et al. randomized patients to either undergo gradual weaning vs proceed to clamping within 24 hours. No differences in the incidence of hydrocephalus or shunt requirements were noted [17]. Rao., et al. retrospectively reviewed outcomes before and after an institutional change from gradual to rapid weaning, though this was performed in conjunction with draining CSF intermittently instead of constantly. The intermittent/ rapid group had lower shunt placement rates, shorter EVD durations, and shorter hospital and ICU lengths of stay. Intriguingly, the rates of EVD catheter occlusion were also lower with intermittent drainage and rapid weaning [25]. It is unclear which of the interventions was responsible for these outcomes, and prospective validation will be required. However, these studies offer proof-ofprinciple that rapid weaning may be safe in selected patients.

\section{EVD care bundles}

Care bundles refer to standardized practices and protocols for EVD insertion and management. The primary goal is to reduce complications such as infection. Bundles have been adopted by many institutions and may contain a variety of practices including standardized insertion technique, prophylactic antibiotic use, impregnated catheter use, limitations on CSF sampling/catheter access, sterile dressing use and other workflows [12]. Although randomized studies are lacking, prospective studies following bundle implementation have documented significant decreases in infec- 
tion rate [27]. However, because bundles by definition comprise multiple practices, it may be unclear which specific changes are responsible for improved outcomes [14]. Bundle implementation also serves to bring more attention to practices already in place and create a 'culture of safety' that may improve outcomes independent of specific interventions [19].

Bundles should at a minimum include standardized insertion technique, antibiotic prophylaxis, CSF sampling limits, catheter access regulations, sterile dressing practices and weaning protocol. Providers seeking to formulate evidence-based bundles may refer to the Neurocritical Care Society consensus statement on EVDs [12], as well as guidelines from the Brain Trauma Foundation [6], Infectious Diseases Society of America [29] and Centers for Disease Control [15]. However, studies published after each guideline statement should also be critically evaluated.

\section{Conclusion}

EVDs are among the most commonly performed neurosurgical procedures. Management of patients with EVDs requires collaboration between teams of diverse specialists. The evidence for myriad practices surrounding EVD insertion and management remains limited in quality and quantity. We provide a unique review of the considerations surrounding EVD insertion, complications and management. Our goal is to make these concepts accessible to a broad audience and introduce the critical themes and terminology necessary to formulate interdisciplinary care plans.

\section{Author Contributions}

All authors participated in the conception, design, collection of data, analysis and interpretation of data, drafting the article and critically revising the article. All authors reviewed the submitted version of the manuscript.

\section{Funding}

None.

\section{Competing Interests and Disclosures}

The authors have no financial disclosures to declare. The authors emphasize that the contents of this work are not meant to provide definitive clinical management protocols. Safe performance of procedures discussed herein must be tailored to each patient's circumstances, following careful judgment from treating physicians and adhering to institution- and location-specific guidelines. The views expressed in this work exclusively reflect impressions from the authors' own individual experiences and not those of any department or institution. This work does not reflect the official position of the journal or any of its editors.

\section{Bibliography}

1. Abbott NJ. "Evidence for bulk flow of brain interstitial fluid: significance for physiology and pathology". Neurochemistry International 45 (2004): 545-552.

2. Arabi Y., et al. "Ventriculostomy-associated infections: incidence and risk factors". American Journal of Infection Control 33 (2005): 137-143.

3. Bauer DF., et al. "The relationship between INR and development of hemorrhage with placement of ventriculostomy". The Journal of Trauma 70 (2011): 1112-1117.

4. Binz DD., et al. "Hemorrhagic complications of ventriculostomy placement: a meta-analysis". Neurocritical Care 10 (2009): 253-256.

5. Blomstedt GC. "Results of trimethoprim-sulfamethoxazole prophylaxis in ventriculostomy and shunting procedures. A double-blind randomized trial". Journal of Neurosurgery 62 (1985): 694-697.

6. Carney N., et al. "Guidelines for the Management of Severe Traumatic Brain Injury, Fourth Edition". Neurosurgery 80 (2017): 6-15.

7. Chan KH and Mann KS. "Prolonged therapeutic external ventricular drainage: a prospective study". Neurosurgery 23 (1988): 436-438.

8. Changa AR., et al. "Management of Elevated Intracranial Pressure: a Review". Current Neurology and Neuroscience Reports 19 (2019): 99.

9. Chau CYC., et al. "The Evolution of the Role of External Ventricular Drainage in Traumatic Brain Injury". Journal of Clinical Medicine Research 8 (2019).

10. Dey M., et al. "Bleeding and infection with external ventricular drainage: a systematic review in comparison with adjudicated adverse events in the ongoing Clot Lysis Evaluating Accelerated Resolution of Intraventricular Hemorrhage Phase III (CLEAR-III IHV) trial". Neurosurgery 76 (2015): 291-300.

11. Dorresteijn K., et al. "Factors and measures predicting external CSF drain-associated ventriculitis: A review and meta-analysis". Neurology 93 (2019): 964-972.

12. Fried HI., et al. "The Insertion and Management of External Ventricular Drains: An Evidence-Based Consensus Statement: A Statement for Healthcare Professionals from the Neurocritical Care Society". Neurocritical Care 24 (2016): 61-81. 
13. Hagel S., et al. "External ventricular drain infections: risk factors and outcome". Interdisciplinary Perspectives on Infectious Diseases (2014): 708531.

14. Harrop JS., et al. "Impact of a standardized protocol and antibiotic-impregnated catheters on ventriculostomy infection rates in cerebrovascular patients". Neurosurgery 67 (2010): 187-191.

15. Horan TC., et al. "CDC/NHSN surveillance definition of health care-associated infection and criteria for specific types of infections in the acute care setting". American Journal of Infection Control 36 (2008): 309-332.

16. Kakarla UK., et al. "Safety and accuracy of bedside external ventricular drain placement". Neurosurgery 63 (2008): 162166.

17. Klopfenstein JD., et al. "Comparison of rapid and gradual weaning from external ventricular drainage in patients with aneurysmal subarachnoid hemorrhage: a prospective randomized trial”. Journal of Neurosurgery 100 (2004): 225-229.

18. Korinek AM., et al. "Prevention of external ventricular drain-related ventriculitis". Acta Neurochirurgica (Wien) 147 (2005): 39-45.

19. Kubilay Z., et al. "Decreasing ventricular infections through the use of a ventriculostomy placement bundle: experience at a single institution". Journal of Neurosurgery 118 (2013): 514-520.

20. Lozier AP., et al. "Ventriculostomy-related infections: a critical review of the literature". Neurosurgery 62.2 (2008): 688-700.

21. Mayhall CG., et al. "Ventriculostomy-related infections. A prospective epidemiologic study". The New England Journal of Medicine 310 (1984): 553-559.

22. Omar MA and Mohd Haspani MS. "The risk factors of external ventricular drainage-related infection at hospital kuala lumpur: an observational study". Malaysian Journal of Medical Sciences 17 (2010): 48-54.

23. Poon WS., et al. "CSF antibiotic prophylaxis for neurosurgical patients with ventriculostomy: a randomised study". Acta Neurochirurgica 71 (1998): 146-148.

24. Pople I., et al. "Comparison of infection rate with the use of antibiotic-impregnated vs standard extraventricular drainage devices: a prospective, randomized controlled trial". Neurosurgery 71 (2012): 6-13.
25. Rao SS., et al. "Intermittent CSF drainage and rapid EVD weaning approach after subarachnoid hemorrhage: association with fewer VP shunts and shorter length of stay". Journal of Neurosurgery (2019): 1-6.

26. Sussman ES., et al. "Hemorrhagic complications of ventriculostomy: incidence and predictors in patients with intracerebral hemorrhage". Journal of Neurosurgery 120 (2014): 931-936.

27. Talibi SS., et al. "The implementation of an external ventricular drain care bundle to reduce infection rates". British Journal of Neurosurgery (2020): 1-6.

28. Tubbs RS., et al. "The foramen of Monro: a review of its anatomy, history, pathology, and surgery". Child's Nervous System 30 (2014): 1645-1649.

29. Tunkel AR., et al. "Infectious Diseases Society of America's Clinical Practice Guidelines for Healthcare-Associated Ventriculitis and Meningitis". Clinical Infectious Diseases 64 (2017): e34-e65.

30. Zabramski JM., et al. "Efficacy of antimicrobial-impregnated external ventricular drain catheters: a prospective, randomized, controlled trial". Journal of Neurosurgery 98 (2003): 725730.

\section{Assets from publication with us}

- Prompt Acknowledgement after receiving the article

- Thorough Double blinded peer review

- Rapid Publication

- Issue of Publication Certificate

- High visibility of your Published work

Website: www.actascientific.com/

Submit Article: www.actascientific.com/submission.php Email us: editor@actascientific.com

Contact us: +919182824667 